\title{
Design, Manufacturing and Test of a High-Precision MEMS Inclination Sensor for Navigation Systems in Robot-Assisted Surgery
}

\author{
Benjamin Arnold ${ }^{1, ~ *, ~ D a n i e l ~ W o h l r a b ~}{ }^{1}$, Christoph Meinecke ${ }^{2}$, Danny Reuter $^{2}$, Jan Mehner $^{1}$ \\ ${ }^{1}$ Department of Microsystems and Biomedical Engineering, Faculty of Electrical Engineering and Information Technology, University of \\ Technology Chemnitz, Chemnitz, Germany \\ ${ }^{2}$ Center for Microtechnologies, University of Technology Chemnitz, Chemnitz, Germany
}

\section{Email address:}

benjamin.arnold@etit.tu-chemnitz.de (B. Arnold), daniel.wohlrab@etit.tu-chemnitz.de (D. Wohlrab),

meinecke@zfm.tu-chemnitz.de (C. Meinecke), danny.reuter@zfm.tu-chemnitz (D. Reuter), jan.mehner@etit.tu-chemnitz.de (J. Mehner)

${ }^{*}$ Corresponding author

\section{To cite this article:}

Benjamin Arnold, Daniel Wohlrab, Christoph Meinecke, Danny Reuter, Jan Mehner. Design, Manufacturing and Test of a High-Precision MEMS Inclination Sensor for Navigation Systems in Robot-Assisted Surgery. International Journal of Biomedical Science and Engineering. Vol. 6, No. 1, 2018, pp. 1-6. doi: 10.11648/j.ijbse.20180601.11

Received: November 29, 2017; Accepted: January 18, 2018; Published: February 1, 2018

\begin{abstract}
Robot supported minimally invasive interventions are state of the art in operating theatres. To increase the accuracy of surgical instrument positioning, high-precision motion tracking systems are required. The miniaturization of microelectromechanical systems (MEMS) facilitates the placing of orientation detection sensors close to the mounting of the surgical instrument to enhance positioning accuracy. A high resolution inclination sensor was developed using the innovative approach of laser-micro-welding. Trench sizes down to $800 \mathrm{~nm}$ are fabricated with more than 6-fold increase in aspect ratios (structure depth to electrode gap) compared to sensors without gap reduction. Electrical and physical tests as well as finiteelement-simulations were performed. An increased sensitivity from $7.2 \mathrm{fF} /{ }^{\circ}$ up to $60 \mathrm{fF} /{ }^{\circ}$ was verified for the sensor with reduced electrode gap and a customized ASIC.
\end{abstract}

Keywords: Surgical Robot Navigation, Inertial Sensor, Inclination Sensor, Microelectromechanical System (MEMS), Gap Reduction

\section{Introduction}

In the past few years surgical robots firmly established to support surgeons in minimally invasive interventions. One benefit of the usage of robots in medicine is the ergonomical posture of the surgeon that eases high concentrated operating especially over a long time. Additionally the physical presence of high specialized surgeons in the operating theatre is technically no longer necessary since 2002. This was demonstrated by New York surgeons who realized a remote robot-assisted laparoscopic removal of the gallbladder of a patient in Strasbourg with the ZEUS System (Computer Motion, Galeta, CA) [1]. In 2013, about $85 \%$ of radical laparoscopic prostatectomies were realized robot-assisted in the USA and it is foreseeable the rate will still increase [2].

Fields of application beside the biopsy and resection of tumours are the locally limited high-dose irradiation, brain and abdominal surgery as well as cardiac surgery, in summary all precise navigation tasks. Major advantages of these systems are the possibility to scale down the hand movement of the surgeon to high-accuracy fine pitched motions of the surgical instruments, the precise locking mechanism and tremor cancellation of the surgeon's steering movements [3]. Consequently, unintentional injury of healthy tissue, blood vessels or nerves can be prevented.

\section{Aim}

To achieve very accurate instrument positioning, highprecision motion tracking systems are essential to get for example the actual tilt of an endoscope. Due to miniaturization of MEMS, the placing of orientation 
detection sensors close to the endoscope is possible. Furthermore, the integration of MEMS tilt sensors into the surgical instrument near the mounting can facilitate the measurement of vibrations and their compensation. The developed capacitive MEMS sensor complies with the requirements of small size and high-precision inclination measurement.

\section{Methods}

\subsection{Fabrication Technique}
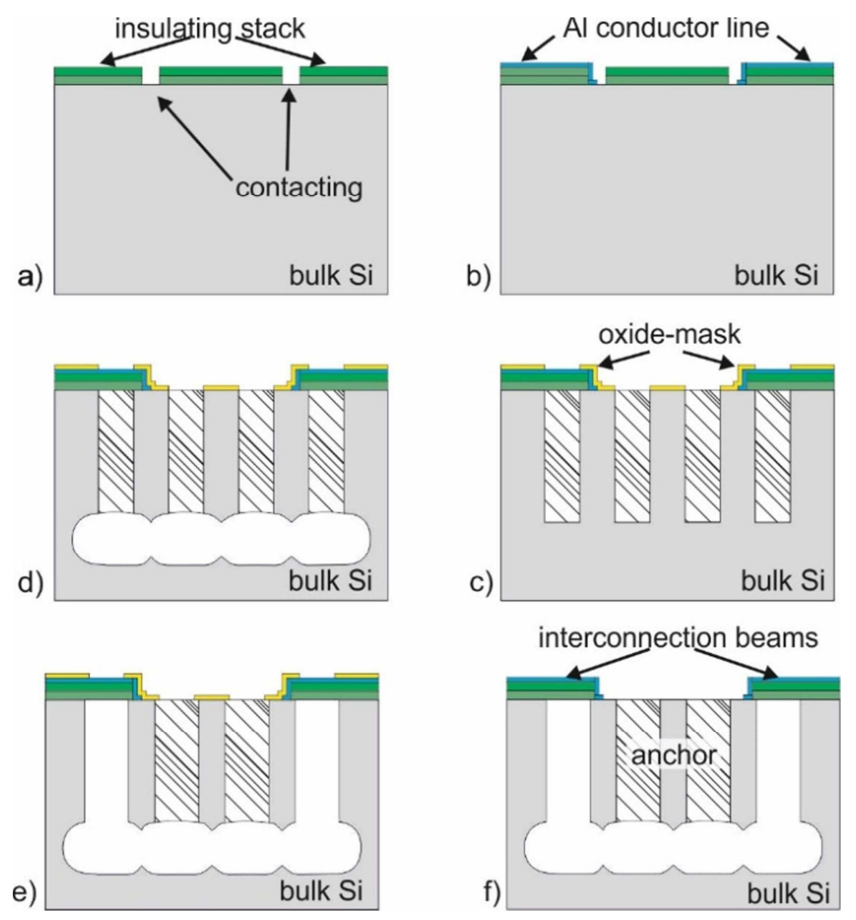

Figure 1. Schematic process flows of the technology: a) deposition and patterning of insulating layers b) deposition and patterning of conducting layers c) DRIE of silicon d) release etching of silicon e) isotropic etching for release of interconnection beams f) removal of oxide mask.

The sensor is fabricated by using silicon high aspect ratio micromachining (HARM) technology to achieve $50 \mu \mathrm{m}$ thick structures with about $4.5 \mu \mathrm{m}$ trench size [4]. The performed process flow of the technology is presented in Figure 1. This technology starts with the deposition and the patterning of the insulation layer on highly p-doped single crystalline SiWafer (a). Subsequently, aluminium is sputtered and patterned as interconnection layer (b) Afterwards the silicon microstructures were etched via a silicon dioxide mask in a STS Multiplex ICP System by deep reactive ion etching (DRIE) (c). The deposition of the passivation layer, the spacer etching, and the isotropic etching for releasing the movable structures is performed in situ with the DRIE (d). After removal of the sidewall passivation, the AIM specific interconnection beams were undercut by an isotropic etching process (e). Finally the silicon dioxide layer used as the mask for DRIE is removed (f). Due to the isotropic plasma etch step after the DRIE the electrode gap is widened, which affect the aspect ratio (structure depth to electrode gap) and thereby the sensitivity of the MEMS. The sensitivity of capacitive inertial sensors is highly influenced by the capacitance gradient due to an electrode movement. To increase the capacitive gradient, the aspect ratio of the vertical comb electrodes should be increased. However, fabrication restrictions limit the minimum distance between the electrodes. Therefore, the reduction of the trench width after patterning of the microstructures has been proposed and revealed. The fabrication of sub-micron trenches [5-10] or structures with very high aspect ratios up to 100 [6] is advantageous for many applications. To increase the aspect ratio of the sensing electrode gap, different technologies for reduction of trench width of capacitive micro structures below the technological limitations have been proposed and demonstrated in recent years [11-15].

For post-process technologies electrostatically [11, 13] and thermally [12] actuated gap reduction mechanisms have been presented. The permanent application of a voltage for locking the mechanism in its final position is not energy-efficient and possibly influences the transducer signals. Using ratchet structures or bistable mechanisms for locking the reduced gap are critical for the reliability of the transducers. An inprocess gap reduction approach [14] utilizes residual stress in microstructures, coated with silicon dioxide. However, the technique causes a high temperature sensitivity of the microstructures. Furthermore the silicon micro-welding process [15] seems to be reliable but has a need of large mechanisms, which enlarges the chip size dramatically. In this work a high-resolution tilt sensors was developed, utilizing the innovative approach of laser-micro-welding [16], to enable high aspect ratios up to 100 for trench sizes down to $800 \mathrm{~nm}$ after fabrication. The novelty is due to the use of a differential capacitor design, which greatly complicates the reduction of the electrode gap, but with the advantage of higher linearity and sensitivity compared to single capacitor.

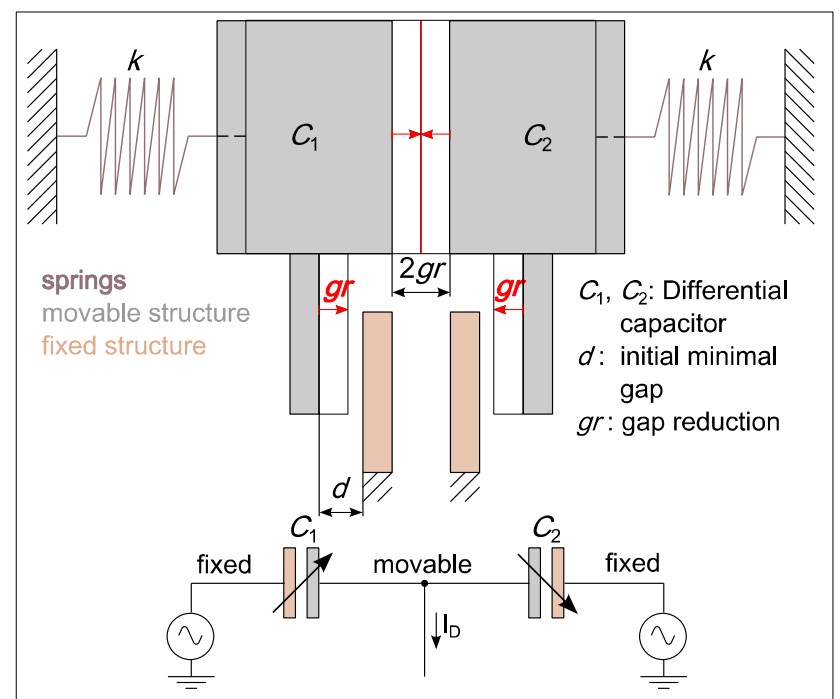

Figure 2. Schematic picture of MEMS differential capacitor working principle with gap-reduction mechanism and equivalent circuit. 
A post process gap reduction mechanism, based on the separation of the seismic mass into two symmetrical parts (Figure 2) was integrated in the MEMS layout. After all critical MEMS process steps the two initially separated sides of the differential capacitor were connected by using the electrostatic force of the sense comb electrodes (Figure 3). In initial position the two separated seismic masses have an electrode gap of about $4.5 \mu \mathrm{m}$, which can be easily fabricated using standard DRIE etching processes. The electrostatic force necessary to connect the masses is generated by a small mismatch of the gaps. The fixation was achieved by adhesive bonding of overhanging aluminum layers in the bonding zone and partly welding due to a briefly high current. Long-term stability and reliability after the first fixation step was ensured by laser-micro-welding on the aluminium layers (see Figure 4). For the welding process was performed on a laser micro treatment facility [17], consisting of a femtosecond laser and a galvanometer scan system for high accuracy beam deflection and focusing.
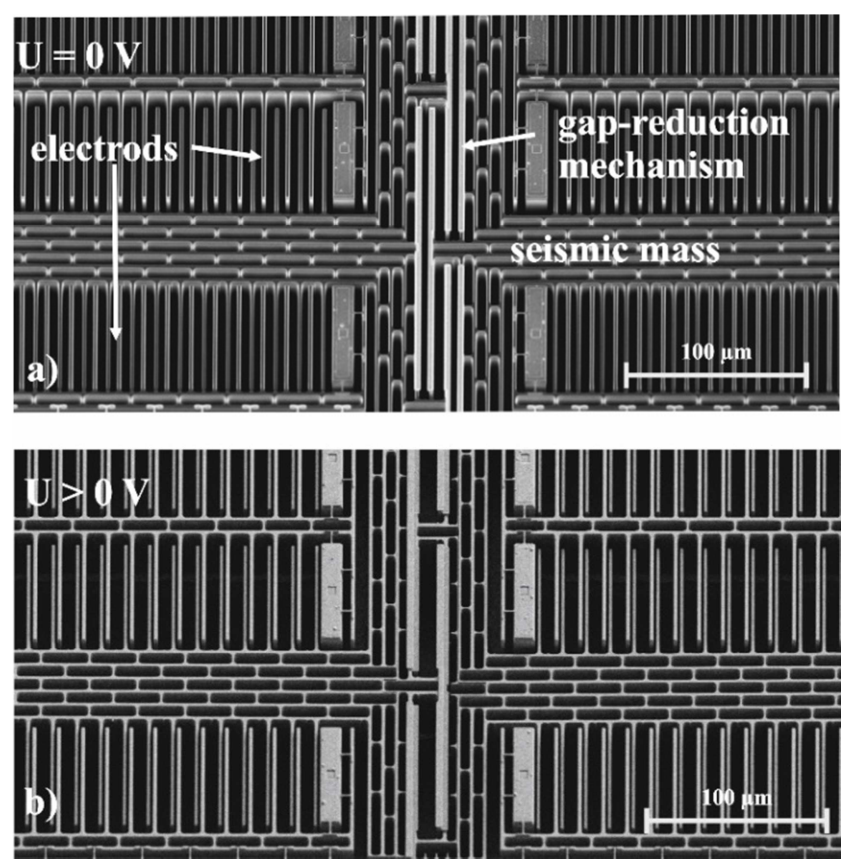

Figure 3. SEM picture of the AIM-MEMS with the gap reduction mechanism. a) without pull-in voltage (initial gap) and b) with applied pullin voltage, which results in minimized electrode gaps and connected seismic masses.

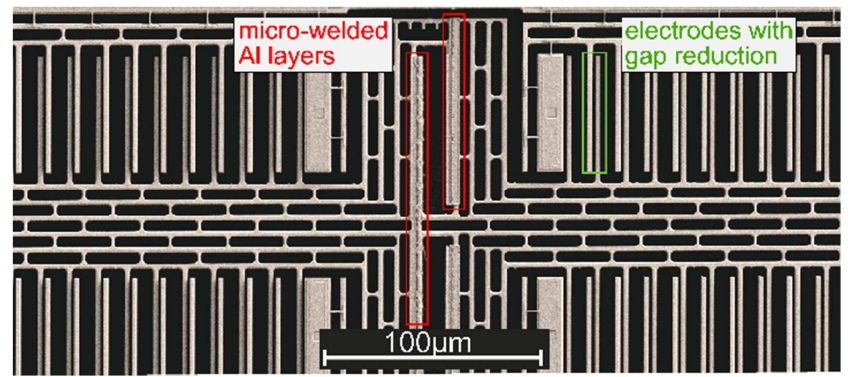

Figure 4. SEM image of the laser-micro-welded area and the reduced electrode gaps of the tilt sensor.

\subsection{Sensor Design}

To determine design parameters finite element (FE) simulations were implemented especially taken the gap reduction process into account. Therefore, an ANSYS ${ }^{\circledR}$ FE full model perturbation analysis was performed to design the mechanical system considering the preload of the springs due to the gap reduction mechanism. In Figure 5 the full mechanical FE model including all components like electrodes, anchor mass and spring is presented.

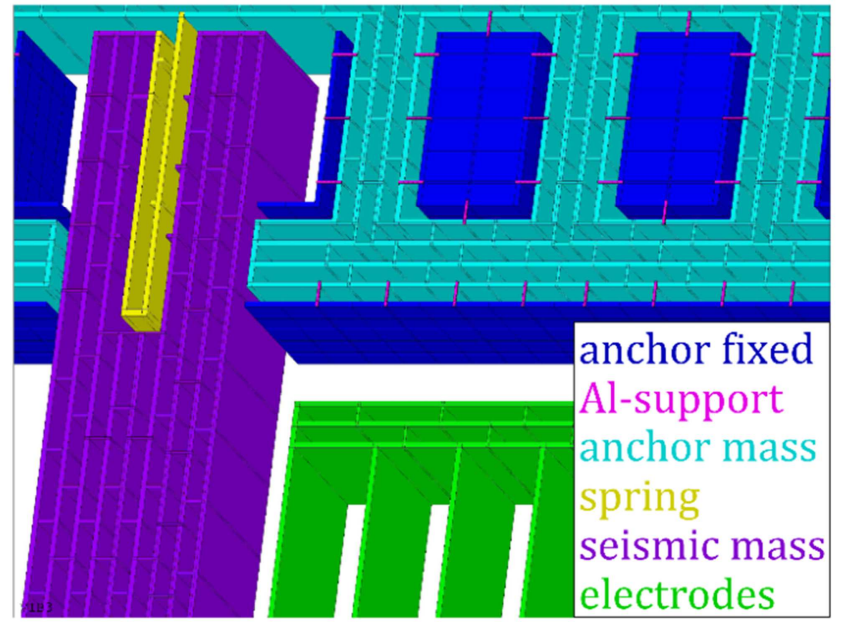

Figure 5. Component view of the full mechanical FE model of the sensor structure.

A design study of the electrode combs has been done using parameterized Computational Fluid Dynamics (CFD) simulations to optimize the damping behaviour for the 800 $\mathrm{nm}$ reduced gap. Input parameters of the CFD model were the ambient air pressure $p$, which highly influences the damping coefficient of the system, as well as the oscillation amplitude $s$ and the electrode overlap length. The pressure range is $1 \mathrm{kPa}$ to $100 \mathrm{kPa}$ due to the possibility of evacuating the encapsulated system. The oscillation amplitude $s$ can be set up to a maximum of $300 \mathrm{~nm}$. The damping coefficient was determined on several interpolation points with the help of CFD simulations of a single electrode comb cell. Considering the entire comb capacitor, translational periodicity conditions were used on the outer walls where the air can stream from one cell to another together with a simple multiplication of the damping coefficient with the total number of comb cells. The interpolation points are fitted with the least-square method within a Matlab $^{\circledR}$ script to the polynomial function $b(s, p)$ of the damping constant. In Figure 6 the result of the fit for an overlap length of the electrodes of $10 \mu \mathrm{m}$ can be seen.

The damping coefficient from CFD was used in a harmonic FE analysis on the full mechanical sensor model to estimate the upper and lower design limits resulting from fabrication technology tolerances (see Figure 7). 


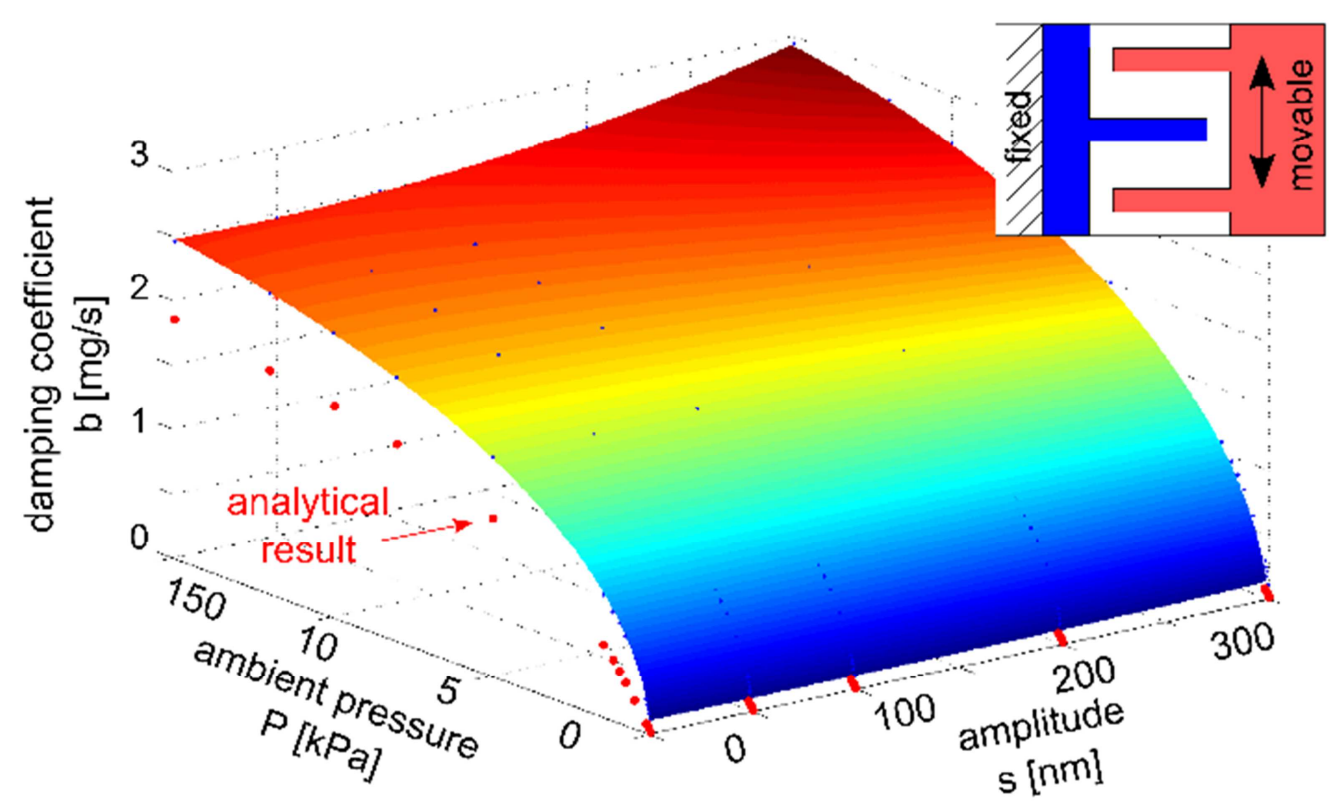

Figure 6. Damping coefficient from parameterized CFD model of comb electrodes for 10 um overlap.

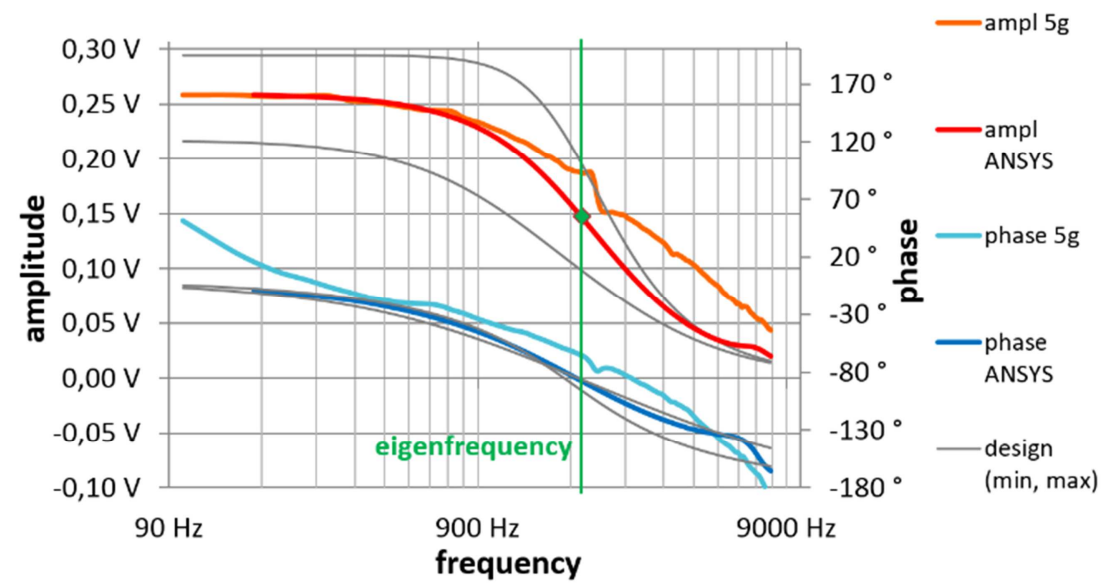

Figure 7. Sensor amplitude and phase response for a $5 g$ excitation in comparison to simulation with upper and lower design limits due to fabrication tolerances.

\section{Results}

All Measurements on the performance of the entire sensor system were carried out on the test setup shown in Figure 8. Stacking of the ASIC and sensor chips was used on top of a thermoplastic thermal compensation structure designed to minimize the stress impact due to a temperature gradient and thus minimize the thermal drift of the device.

Using the post-process technology of laser-micro-welding, the aspect ratio of the electrode gaps is increased from initially 15 to 90 . Furthermore, the stability and the reliability of the welded connections were confirmed by a long-term analysis.

Results of the parameterized CFD analysis of the comb electrode show that the damping coefficient is underrated by at least $30 \%$ compared to analytical methods [18], which emphasizes the importance of the damping simulations (see Figure 6). This is due to the fact that the analytical equations are only valid for relatively large gaps where continuum conditions can be assumed, but not for sub-micron gaps.

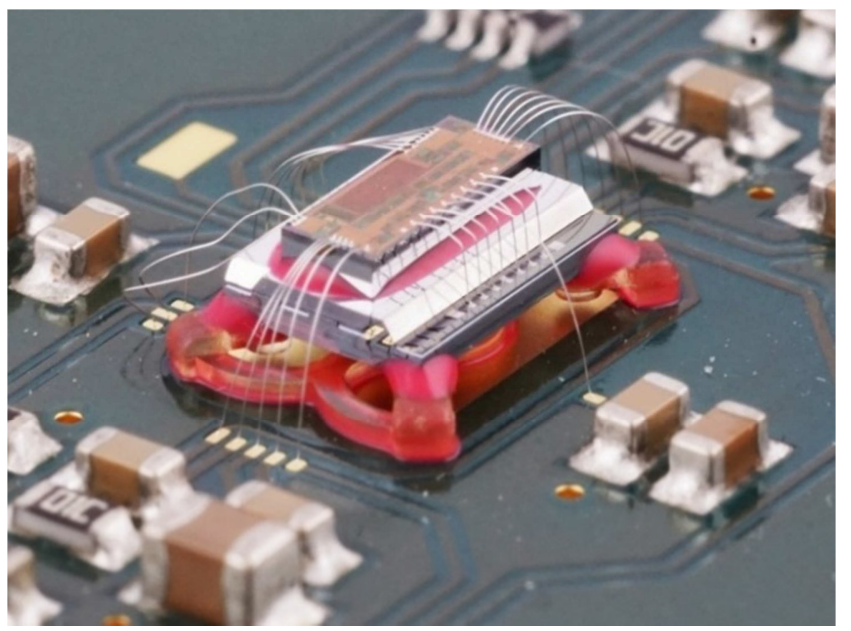

Figure 8. Test setup with ASIC-sensor-chip stack and thermal compensation structure on circuit board. 
Electrical and physical tests of the sensor without gap reduction were performed. The differential capacitance over tilt angle $\alpha$ was measured in a range from $-40^{\circ}$ to $40^{\circ}$ (see Figure 9). Due to the measurements the sensitivity of the sensor without gap reduction is with $7.2 \mathrm{fF} /{ }^{\circ}$ lower compared to the calculated value from the first design. A uniform structure thickness is achieved by thinning of the active wafer before patterning. The nominal thickness value used in the design was $50 \mu \mathrm{m}$, though the characterization of the processed structure shows that the thickness after thinning is only around $40 \mu \mathrm{m}$ which leads to a lower sensitivity and fits to the simulations. Besides the effect of linearization around the angle $\alpha=0^{\circ}$, another advantage of the differential capacitor is the increase in sensitivity by the factor of 2 compared to single capacitor arrangements.

With the matched design parameters the design procedure could be validated and a sensitivity of at least $60 \mathrm{fF} /{ }^{\circ}$ for the improved sensor with gap reduction can be proposed (see Figure 10). Compared to real sensor data the maximum error of the amplitude response up to the working range of $1 \mathrm{kHz}$ is only around 1\% (see Figure 7).

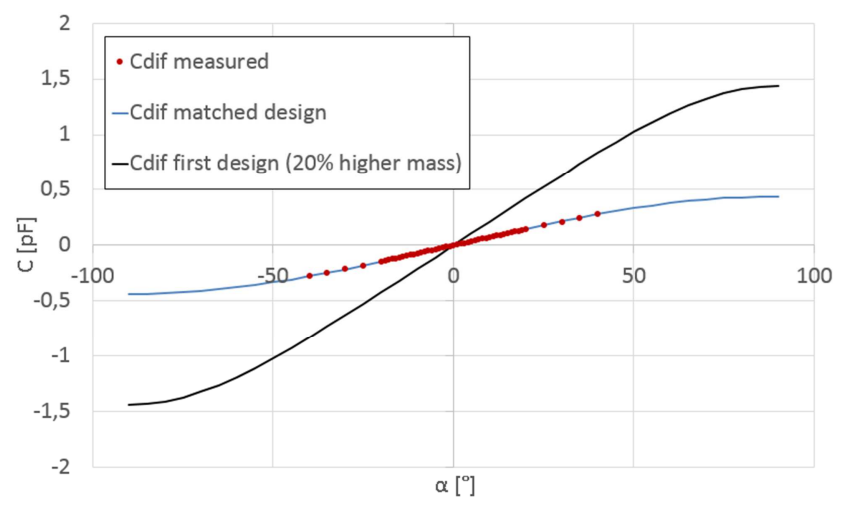

Figure 9. Measured and simulated differential capacitance over tilt angle for sensor without gap reduction.

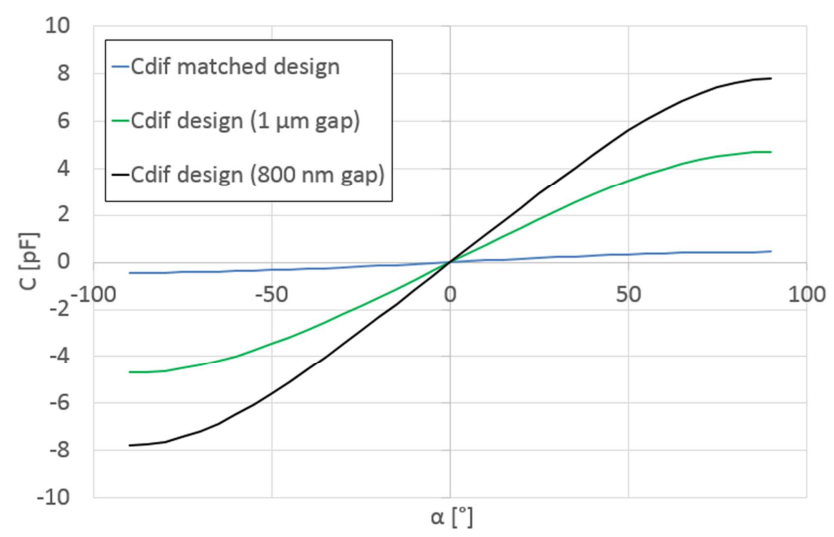

Figure 10. Comparison of differential capacitance over tilt angle with and without gap reduction with matched design parameters.

\section{Conclusion}

In this paper, a high-precision capacitive MEMS tilt sensor was designed for application in precise orientation measurements. The inclination sensor was fabricated using laser-micro-welding and a gap reduction mechanism on differential capacitor arrangement to improve the sensitivity of the device. Measurements on the real system verify the developed FEM design model. Scheduled measurements on the sensor with reduced gap are outstanding but very promising. The sensitivity of the device can be improved by a factor of at least 8 compared to a standard sensor without gap reduction, making it well suitable for highly accurate navigation tasks in robot-assisted surgery.

\section{Acknowledgements}

The authors thanks the staff of Center for Microtechnologies of the TU Chemnitz, Laserinstitut Hochschule Mittweida and Fraunhofer Institute ENAS. The German Federal Ministry of Education and Research (BMBF) funded this work within the joint project GIPRIS (fund number 16M3094D). The responsibility for the content of this publication lies with the author.

\section{References}

[1] J. Marescaux, J. Leroy, F. Rubino et al. Transcontinental Robot-Assisted Remote Telesurgery: Feasibility and Potential Applications. Annals of Surgery. Vol. 235, No. 4, 2002, pp. 487-492.

[2] The Lancet. Robotic surgery evaluation: 10 years too late. The Lancet. Vol. 388, Iss. 10049, 2016, p. 1026, DOI 10.1016/S0140-6736 (16) 31586-0.

[3] K. J. Rebello. Applications of MEMS in surgery. Proceedings of the IEEE. Vol. 92, Iss. 1, 2004, pp. 43-55, DOI 10.1109/JPROC.2003.820536.

[4] A. Bertz, M. Küchler, R. Knöfler, T. Gessner. A novel high aspect ratio technology for MEMS fabrication using standard silicon wafers. Sensors and Actuators A: Physical. Vol. 97-98, 2002, pp. 691-701, DOI 10.1016/S0924-4247 (02) 00006-7.

[5] R. Abdolvand, F. Ayazi. An advanced reactive ion etching process for very high aspect-ratio sub-micron wide trenches in silicon. Sensors and Actuators A: Physical. Vol. 144, Iss. 1, 2008, pp. 109-116, DOI 10.1016/j.sna.2007.12.026.

[6] F. Marty, L. Rousseau, B. Saadany et al. Advanced etching of silicon based on deep reactive ion etching for silicon high aspect ratio microstructures and three-dimensional micro- and nanostructures. Microelectronics Journal. Vol. 36, Iss. 7, 2005, pp. 673-677, DOI 10.1016/j.mejo.2005.04.039.

[7] S. Tachi, K. Tsujimoto, S. Okudaira. Low-temperature reactive ion etching and microwave plasma etching of silicon. Applied Physics Letters. Vol. 52, Iss. 8, p. 616, DOI 10.1063/1.99382.

[8] F. Lärmer, A. Schilp. Method of anisotropically etching silicon. Patent US 5501893, 1996.

[9] B. Wu, A. Kumar, S. Parmarthy. High aspect ratio silicon etch: A review. Journal of Applied Physics. Vol. 108, Iss. 5, 051101, 2010, DOI 10.1063/1.3474652. 
[10] S. D. Senturia. CAD challenges for microsensors, microactuators, and microsystems. Proceedings of the IEEE. Vol. 86, Iss. 8, 1998, pp. 1611-1626, DOI 10.1109/5.704266.

[11] D. Galayko et al. Design, realization and testing of micromechanical resonators in thick-film silicon technology with postprocess electrode-to-resonator gap reduction. Journal of Micromechanics and Microengineering. Vol. 13, 2004, pp. 134-140.

[12] C. Acar et al. Post-Release Capacitance Enhancement in Micromachined Devices. IEEE Sensors, Vienna, Austria, Oct. 24-27, 2004, pp. 268-271, DOI 10.1109/ICSENS.2004.1426153.

[13] W.-C. Chen et al. Realizing deep-submicron gap spacing for CMOS MEMS resonators with frequency tuning capability via modulated boundary conditions. IEEE International Conference on MEMS, Hong Kong, China, Jan. 24-28, 2010, pp. 735-738, DOI 10.1109/MEMSYS.2010.5442301.

[14] D. Reuter et al. In-Process Gap Reduction of Capacitive Transducers. Sensors and Actuators A: Physical. Vol. 126, Iss. 1, 2006, pp. 211-217, DOI 10.1016/j.sna.2005.09.033.
[15] M. Nowack et al. Novel Post-Process Gap Reduction Technology of High Aspect Ratio Microstructures Utilizing Micro Welding. Transducers Conference, Beijing, China, Jun. 5-9, 2011, pp. 1352-1355, DOI 10.1109/TRANSDUCERS.2011.5969505.

[16] Meinecke $\mathrm{C}$ et al. Micro welding of aluminum for post process electrode gap reduction using femtosecond laser. Transducers Conference, Anchorage, Alaska, USA, Jun. 2125, 2015, pp. 1354-1357, DOI 10.1109/TRANSDUCERS.2015.7181183.

[17] J. Schille et al. Highspeed Laser Micro Processing using Ultrashort Laser Pulses. Journal of Laser Micro / Nanoengineering. Vol. 9, No. 2, 2014, pp. 161-168, DOI 10.2961/jlmn.2014.02.0015.

[18] T. Veijola. Compact models for squeezed-film dampers with inertial and rarefied gas effects. Journal of Micromechanics and Microengineering. Vol. 14, 2004, pp. 1109-1118. 